\title{
A GLOBAL EQUILIBRIUM AS THE FOUNDATION OF QUANTUM RANDOMNESS
}

\author{
Detlef Dürr,,${ }^{1,2}$ Sheldon Goldstein ${ }^{1}{ }^{\text {And Nino Zanghí }}{ }^{1,3}$
}

\begin{abstract}
We analyze the origin of quantum randomness within the framework of a completely deterministic theory of particle motion-Bohmian mechanics. We show that a universe governed by this mechanics evolves in such a way as to give rise to the appearance of randomness, with empirical distributions in agreement with the predictions of the quantum formalism. Crucial ingredients in our analysis are the concept of the effective wave function of a subsystem and that of a random system. The latter is a notion of interest in its own right and is relevant to any discussion of the role of probability in a deterministic universe.
\end{abstract}

\section{INTRODUCTION}

Nonequilibrium is an essential aspect of our universe. However, as it is well known, no local physical explanation can fully account for it. As R. Penrose [15] has colorfully emphasized, the explanation of local thermodynamic laws requires an appeal to appropriate cosmological conditions, namely, very improbable initial conditions corresponding to a global low entropy initial state of the universe.

Here we wish to explain that quantum laws also require a cosmological setting, and that they are indeed founded on a global equilibrium for a level of description

\footnotetext{
${ }^{1}$ Department of Mathematics, Rutgers University, New Brunswick, New Jersey 08903.

${ }^{2}$ Current address: Fakultät für Mathematik, Universität München, 8000 München 2, Germany.

${ }^{3}$ Current address: Istituto di Fisica, Università di Genova, INFN, 16146 Genova, Italy.
} 
beneath thermodynamic nonequilibrium. Our analysis shows that quantum randomness, as, for example, embodied in Born's statistical law, should be regarded as a local manifestation of a global quantum equilibrium state of our universe.

However, orthodox quantum theory is inadequate for the very formulation of such a contention. In fact, on the cosmological level there is no observer outside the system to perform measurements on it. In orthodox quantum theory only the appeal to the observer gives physical significance to the theoretical entities - such as the wave function - entering into the formalism.

Suppose we do seek an observer-free (formulation of) quantum theory without unpleasant metaphysical implications $[\mathbf{1 0}]$ or drastic modifications of the mathematical structure of the orthodox theory [12]. How are we to proceed? First, we should be clear as to what precisely the quantum formalism is about! What we are usually told, and what we believe is correct, is that the quantum formalism is merely a measurement formalism.

Indeed, the most modest attitude one could adopt then towards orthodox quantum theory would appear to be that of regarding it as a phenomenological formalism, roughly analogous to the thermodynamic formalism, for the description of certain macroscopic regularities. However, the thermodynamic formalism can be derived from microscopic physics, from the behavior of the constituents of the macroscopic systems. Should we not then demand a similar account of the quantum formalism?

Note that in the absence of such an account, the quantum formalism itself suffers from serious vagueness and ambiguity, owing to the fact that this formalism seems to refer to the relationship between the microscopic and the macroscopic, for example insofar as it refers to "measurements of observables" for microscopic systems - and in practice this is what it always does! What is meant by the "mea- 
surement" of such an "observable," or, more generally, what is "measurement"? Is not something like measurement-suitable interactions between systems of interest and their environment-going on, as Bell has emphasized [3], more or less everywhere, all the time? Moreover, as Einstein [13] has emphasized, "on principle, it is quite wrong to try founding a theory on observable magnitudes alone. ...It is the theory which decides what we can observe." Thus, without a fully microscopic theory, the quantum measurement formalism must suffer from a lack of clarity and precision in the statement of its operational details.

Nonetheless, what makes quantum mechanics controversial is not the quantum formalism itself, but rather a further assertion to the effect that we cannot get beneath this formalism, to account for it in microscopic terms. This is, indeed, a radical claim, which in fact can easily be refuted by an explicit "counterexample," the quantum theory of David Bohm [5]. We should recall that the very existence of such a theory has been declared impossible, both physically and mathematically, on the authority of Bohr, of von Neumann, and many others. It is thus all the more remarkable that a counterexample to such claims can easily be obtained, even while ignoring the very formalism with which it is allegedly incompatible.

We have shown in [7] that the requirement of Galilean covariance naturally leads from Schrödinger's equation to a deterministic - though radically non-Newtoniantheory of particles in motion, which we have called Bohmian mechanics (Section 2). We have shown that this theory completely accounts for the phenomenological content of nonrelativistic quantum mechanics $[\mathbf{7}],[\mathbf{9}]$.

Bohmian mechanics is a priori defined only for the universe as a whole, and its applicability to subsystems rests on the concept of the effective wave function, a concept of great importance also for the quantum formalism since it clarifies and 
makes precise the notion of the wave function of a system (Section 4). Nevertheless, Bohmian mechanics eliminates at all scales the paradoxes and perplexities so often associated with orthodox quantum theory.

The second basic concept in our analysis is that of quantum equilibrium, a concept analogous to, but quite distinct from, thermodynamic equilibrium. Quantum equilibrium provides us with a precise and natural notion of typicality: we show that typical initial configurations, for a universe governed by Bohmian mechanics, evolve in such a way as to give rise to the appearance of randomness, with empirical distributions in agreement with the predictions of the quantum formalism (Section $5)$.

There is as yet no satisfactory extension of Bohmian mechanics to the relativistic domain (but see $[\mathbf{8}],[\mathbf{6}],[\mathbf{1}],[\mathbf{4}]$ for some partial results). We wish, however, to point out that the account of quantum randomness which emerges from Bohmian mechanics depends only on rather qualitative features of abstract quantum theorynot on the details of any specific quantum theory such as nonrelativistic quantum mechanics or quantum field theory. Thus, we believe that our results - concerning the status of quantum randomness - have a validity extending beyond the nonrelativistic framework in which they have been derived (Sections 6 and 7).

\section{BOHMIAN MECHANICS}

The element of the quantum formalism which most seems to function as a theoretical entity on the microscopic level, as the objective state, is the wave function. Now suppose that when we talk about the wave function of a system of $N$ particles, we seriously mean what our language conveys, i.e., suppose we insist that "particles" means particles. If so, then the wave function $\psi$ cannot provide a complete 
description of the state of the system; we must also specify its most important feature, the positions of the particles themselves.

For Bohmian mechanics the complete state for a system of $N$ particles is given by

$$
(Q, \psi)
$$

where

$$
Q=\left(\mathbf{Q}_{1}, \ldots, \mathbf{Q}_{N}\right) \in \mathbb{R}^{3 N}
$$

with $\mathbf{Q}_{1}, \ldots, \mathbf{Q}_{N}$ the positions of the particles, and

$$
\psi=\psi(q)=\psi\left(\mathbf{q}_{1}, \ldots, \mathbf{q}_{N}\right)
$$

is the wave function of the system. Note that we use $Q$ for the actual configuration and $q$ for the generic configuration space variable.

As for the time evolution, since $(Q, \psi)$ is indeed the "state," its present specification, say $\left(Q_{0}, \psi_{0}\right)$, must determine the state $\left(Q_{t}, \psi_{t}\right)$ at later times; thus the evolution is defined by first-order differential equations: Schrödinger's equation

$$
i \hbar \frac{\partial \psi_{t}}{\partial t}=H \psi_{t}
$$

for $\psi$, and an evolution equation for $Q$ of the form

$$
\frac{d Q_{t}}{d t}=v^{\psi_{t}}\left(Q_{t}\right)
$$

where

$$
v^{\psi}=\left(\mathbf{v}_{1}^{\psi}, \ldots, \mathbf{v}_{N}^{\psi}\right)
$$


is a vector field on configuration space $\mathbb{R}^{3 N}$. Thus the role of the wave function $\psi$ here is to generate the motion of the particles, through the vector field on configuration space,

$$
\psi \longrightarrow v^{\psi}
$$

to which it is associated.

Moreover, the detailed form of $v^{\psi}$ is determined by requiring space-time symmetryGalilean covariance. This leads rather directly, as the simplest possibility, to

$$
\mathbf{v}^{\psi}=\frac{\hbar}{m} \operatorname{Im} \frac{\nabla \psi}{\psi}
$$

for a one-particle system (note that the $\nabla$ is suggested by rotation invariance, the $\psi$ in the denominator by homogeneity, the Im by time-reversal invariance, and the constant in front is precisely what is required for covariance under Galilean boosts) and to

$$
\mathbf{v}_{k}^{\psi}=\frac{\hbar}{m_{k}} \operatorname{Im} \frac{\nabla_{k} \psi}{\psi}
$$

for many particles.

We've arrived at Bohmian mechanics: For a nonrelativistic system of $N$ particles (for simplicity ignoring spin) the state is given by $(Q, \psi)$ and the evolution by

$$
\begin{aligned}
\frac{d Q_{t}}{d t} & =v^{\psi_{t}}\left(Q_{t}\right) \\
i \hbar \frac{d \psi_{t}}{d t} & =-\sum_{k=1}^{N} \frac{\hbar^{2}}{2 m_{k}} \nabla_{\mathbf{q}_{k}}^{2} \psi_{t}+V \psi_{t}
\end{aligned}
$$

with $v^{\psi}$ given by $(2.1)$ and (2.2). Bohmian mechanics is a fully deterministic theory of particles in motion, but a motion of a highly nonclassical, non-Newtonian 
sort. Moreover, although in orthodox quantum theory the notion of quantum observables as self-adjoint operators plays a fundamental role, while this notion does not appear at all in the formulation of Bohmian mechanics, it can nonetheless be shown that Bohmian mechanics not only accounts for quantum phenomena [5], but also embodies the quantum formalism itself as the very expression of its empirical import $[\mathbf{7}],[\mathbf{9}]$.

In order to arrive at this conclusion, a crucial question which must be addressed is why the familiar ensemble or distribution $\rho=|\psi|^{2}$ plays a distinguished role in Bohmian mechanics. And on the level of mathematics an answer is provided by the concept of equivariance. Consider the ensemble evolution $\rho \rightarrow \rho_{t}$ arising from the Bohmian motion. $\rho_{t}$ is the ensemble to which the Bohmian evolution carries the ensemble $\rho$ in $t$ units of time. If $\rho=\rho^{\psi}$ is a functional of $\psi$ (e.g., $\rho^{\psi}=|\psi|^{2}$ ) we may also consider the transformation $\rho^{\psi} \rightarrow \rho^{\psi_{t}}$ arising from Schrödinger's equation. If these evolutions are compatible,

$$
\left(\rho^{\psi}\right)_{t}=\rho^{\psi_{t}}
$$

we say that $\rho^{\psi}$ is equivariant. In other words, the equivariance of $\rho^{\psi}$ means that under the time evolution it retains its form as a functional of $\psi$.

The distribution $\rho^{\psi}=|\psi|^{2}$ is equivariant. This follows immediately from the observation that the quantum probability current $J^{\psi}=|\psi|^{2} v^{\psi}$, so that the continuity equation

$$
\frac{\partial \rho}{\partial t}+\operatorname{div}\left(\rho v^{\psi}\right)=0
$$

is satisfied by the density $\rho_{t}=\left|\psi_{t}\right|^{2}$. As a consequence,

$$
\text { If } \rho\left(q, t_{0}\right)=\left|\psi\left(q, t_{0}\right)\right|^{2} \text { at some time } t_{0} \text {, then } \rho(q, t)=|\psi(q, t)|^{2} \text { for all } t \text {. }
$$


But what is the physical significance of $\rho=|\psi|^{2}$ ? It turns out that in Bohmian mechanics $\rho=|\psi|^{2}$ reflects the (quantum) equilibrium of the configuration relative to $\psi$ : when a system has wave function $\psi$, its configuration is random, with distribution $|\psi|^{2}$. This assertion can be regarded as roughly analogous to the Gibbs postulate of statistical mechanics: Compare quantum equilibrium

$$
\rho=|\psi|^{2}
$$

whose complete justification [7] in fact turns out to be remarkably easy, with thermodynamic equilibrium

$$
\rho \sim e^{-\beta H}
$$

whose complete justification is remarkably difficult (and as yet nonexistent). We shall expand more on this analogy in the following. First we must elaborate on the status of quantum equilibrium and its empirical consequences.

\section{A DETERMINISTIC UNIVERSE IN QUANTUM EQUILIBRIUM}

The physical meaning of quantum equilibrium involves a crucial subtlety which one can begin to appreciate by first asking the question: Which systems are governed by Bohmian mechanics? The systems which we normally consider are subsystems of a larger system - for example of the universe - whose behavior determines the behavior of its subsystems. Thus, for a Bohmian universe it is only the universe itself which a priori-i.e., without further analysis - can be said to be governed by Bohmian mechanics.

In particular, it is important to recognize that a subsystem cannot in general be governed by Bohmian mechanics, since no wave function for the subsystem need 
exist. This will be so even for free motion - i.e., with no potential in Schrödinger's equation - if the wave function does not properly factorize. For nontrivial potentials the Schrödinger evolution would in any case quickly destroy such a factorization.

Therefore in a universe governed by Bohmian mechanics there is a priori only one wave function, namely that of the universe, as there is a priori only one system governed by Bohmian mechanics, namely the universe itself. Let us consider such a universe. Our first difficulty immediately emerges: In practice $\rho=|\psi|^{2}$ is applied to (small) subsystems. But only the universe has been assigned a wave function. What is meant by the right hand side of $\rho=|\psi|^{2}$ ?

Furthermore, suppose that an initial universal wave function $\Psi_{0}$ has been fixed. Then, since the Bohmian evolution is completely deterministic, once the initial configuration $Q$ of the universe is also specified, all future events, including of course the results of measurements, are determined. Now let $X$ be some subsystem variable - say the configuration of the subsystem at time $t$ - which we would like to be governed by $\rho=|\psi|^{2}$. But how can this possibly be, when there is nothing at all random about $X$ ?

Of course, if we allow the initial universal configuration $Q$ to be random, distributed according to the quantum equilibrium distribution $\left|\Psi_{0}(Q)\right|^{2}$, it follows from equivariance that the universal configuration $Q_{t}$ at later times will also be random, with distribution given by $\left|\Psi_{t}(Q)\right|^{2}$, from which it follows that any variable of interest, for example $X$, has the "right" distribution (see below). However, this is devoid of physical significance! What possible physical significance can be assigned to an ensemble of universes, when we have but one universe at our disposal? We cannot perform the very same experiment more than once. We can perform only 
many similar experiments, differing, however, at the very least, by location or time. In other words, insofar as the use of probability in physics is concerned, what is relevant is not sampling across an ensemble of universes, but sampling across space and time within a single universe. What is relevant is empirical distributions - actual relative frequencies for an ensemble of actual events.

Thus, in order to describe the empirical consequences of quantum equilibrium, two problems must be addressed: that of the meaning of the wave function $\psi$ of a subsystem and that of randomness. It turns out that once we come to grips with the first problem, the question of randomness almost answers itself. We find that $\rho=|\psi|^{2}$ describes the empirical distribution of configurations arising from repetitions of similar experiments, performed at different places or times within a single typical sample of the universe (such as ours), with typicality understood as with respect to universal quantum equilibrium. In other words, we establish the remarkable fact that the observed quantum randomness, as expressed by Born's statistical law, is a simple manifestation of universal quantum equilibrium, in the sense of typicality.

\section{The EFFective Wave FunCtion}

Consider an $N$-particle nonrelativistic universe governed by Bohmian mechanics, with (universal) wave function now denoted by $\Psi$. Focus on a subsystem with generic configuration variables $x$, i.e., on a splitting $q=(x, y)$ where $y$ represents the configuration of the environment of the $x$-system. The actual particle configurations are accordingly denoted by $X$ and $Y$, i.e., $Q=(X, Y)$. Note that $\Psi=\Psi(x, y)$.

How can one assign a wave function to the $x$-system? One obvious possibilityafforded by the existence of the actual configuration - is given by what we call the 
conditional wave function

$$
\psi(x)=\Psi(x, Y) .
$$

(Nonvanishing scalar multiples of wave functions are identified.) Note that by virtue of (2.2) the velocity vector field for the $x$-system is determined by its conditional wave function. This, however, does not in general have the usual dynamical significance of the wave function of a system, as it does not in general evolve according to Schrödinger's equation, even when the $x$-system is dynamically decoupled from its environment.

But one can go further, exploring the form of the universal wave functiona superposition of macroscopically distinct terms - which according to standard quantum measurement theory should arise from a measurement on the $x$-system. One then recognizes that the following definition (see [7]) captures all the desirable phenomenological aspects (through its reference to the "macroscopic"): Suppose that

$$
\Psi(x, y)=\psi(x) \Phi(y)+\Psi^{\perp}(x, y)
$$

where $\Phi$ and $\Psi^{\perp}$ have macroscopically disjoint $y$-supports. If

$$
Y \in \operatorname{supp} \Phi
$$

we say that $\psi$ is the effective wave function of the $x$-system.

The reader familiar with quantum measurement theory should convince himself that our definition of effective wave function coincides with the usual practice of the quantum formalism in ascribing wave functions to subsystems whenever the latter does assign a wave function. In other words, the effective wave function is, in 
effect, a "collapsed" wave function. Note, however, that while in orthodox quantum theory the "collapse" is merely superimposed upon the unitary evolution-without a precise specification of the circumstances under which it may legitimately be invoked - we have now, in Bohmian mechanics, that the evolution of the effective wave function is actually given by a stochastic process - whose probability law is governed by the universal quantum equilibrium distribution - which consistently embodies both unitarity and collapse as appropriate.

\section{From GLOBAL DETERMinism to LOCAL RANDOMNESS}

We wish now to explain how Born's statistical law arises. For this considerfor the moment solely as a mathematical device - the probability space of initial universal configurations $Q$ distributed according to the quantum equilibrium distribution $\mathbf{P}^{\Psi_{0}}(d Q)=\left|\Psi_{0}(Q)\right|^{2} d Q$ - the only natural measure available-where $\Psi_{0}$ is the initial universal wave function. Let $Q_{t}$ and $\Psi_{t}$ denote the universal configuration and the universal wave function at time $t$. Since $\left(Q_{t}, \Psi_{t}\right)$ satisfies the Bohmian evolution (2.3), subject to the initial conditions given by $Q$ and $\Psi_{0}$, it follows that, for given $\Psi_{0}, Q_{t}=\left(X_{t}, Y_{t}\right)$ is a function of the initial universal configuration $Q$ and as such is a random variable.

Note that by equivariance the distribution of $Q_{t}$ is given by $\left|\Psi_{t}\right|^{2}$. It thus follows at once from the definition of the (effective or conditional) wave function that the conditional distribution of the configuration of the $x$-system, given the configuration of its environment, is given by

$$
\mathbf{P}^{\Psi_{0}}\left(X_{t} \in d x \mid Y_{t}\right)=\left|\psi_{t}(x)\right|^{2} d x
$$

where $\psi_{t}$ is the wave function of the $x$-system at time $t$. 
Suppose now that at time $t$ the $x$-system consists itself of many identical subsystems $x_{1}, \ldots, x_{M}$, each one having effective wave function $\psi$ (with respect to coordinates relative to suitable frames). Then the effective wave function of the $x$-system is the product wave function $\psi_{t}(x)=\psi\left(x_{1}\right) \cdots \psi\left(x_{M}\right)$ (see [7]). It follows immediately from (5.1) that, given the configuration $Y_{t}$ of the environment of the $x$-system, the configurations $X_{1}, \ldots, X_{M}$ of the subsystems at time $t$ form a collection of independent random variables, identically distributed, with common distribution $\rho=|\psi|^{2}$. The weak law of large numbers yields then that for large $M$ the empirical distribution of the configurations will be well-approximated by this distribution for the $\left(\mathbf{P}_{Y_{t}}^{\Psi_{0}}\right)$ overwhelming majority of initial universal configurations $Q\left(\right.$ where $\left.\mathbf{P}_{Y_{t}}^{\Psi_{0}}(d Q)=\mathbf{P}^{\Psi_{0}}\left(d Q \mid Y_{t}\right)\right)$. In other words, for typical initial configurations of the universe (consistent with the environment of the $x$-system at time $t$ ) the empirical distribution is given by Born's statistical law.

However, Born's statistical law is supposed to govern not only equal-time ensembles, but also ensembles referring to experiments performed on one or more (sub)systems at possibly different times. Naively, one might think that the construction of an appropriate multitime sample of $M$ subsystems can be obtained by conditioning on the values of the wave functions of these systems at a given sequence of times. However, this procedure ignores a fundamental but surprising aspect of the deterministic evolution of the universe and will in general lead to an unwanted biasing of the sample (see $[7]$ ).

What we must take into account is the fact that, strictly speaking, the times at which our experiments are performed, and indeed the subsystems upon which they are performed, are actually random - not just the results, or the state of the system, 
for any particular experiment, but the time of this experiment as well as the identity of the specific system, of the particular collection of particles, upon which we focus and act. This might appear to be incompatible with the deterministic character of a Bohmian universe, where for each choice of initial universal wave function $\Psi_{0}$ and configuration $Q$ a "history" — past, present and future - is completely determined by these initial conditions. But exactly the opposite is true.

It turns out that upon learning how to deal with this latter problem - of reconciling "local randomness" of the systems upon which experiments are performed with global determinism - we arrive easily at the correct solution to the problem of multitime sampling. The key idea is that of (random) selection:

The selection of a subsystem is characterized by the time $T$ at which the selection occurs and the identity of the selected subsystem. The latter might be described by a projection $\pi$ which realizes a splitting of the universal configuration: $q=$ $(x, y) \equiv\left(\pi q, \pi^{\perp} q\right)$. We must regard both $T$ and $\pi$ as determined by the initial universal configuration, i.e. as random variables - $T$ as a real-valued, and $\pi$ as a projection-valued, function on the space $\mathcal{Q}$ of initial configurations. For $\sigma=(\pi, T)$ we write $X_{\sigma}=\pi Q_{T}$ for the configuration of the system and $Y_{\sigma}=\pi^{\perp} Q_{T}$ for the configuration of its environment.

Moreover, the selection of a subsystem is such that the identity of the particular subsystem and time that it happens to specify are reflected in its environment. In practice, this is expressed by the state of the experimenters, their devices and records, and whatever other features of the environment form the basis of its selection. Thus, for any (nonrandom) $\sigma_{0}=\left(\pi_{0}, t_{0}\right)$, the occurrence of the event $\left\{\sigma=\sigma_{0}\right\}$ must be decided on the basis of the information contained in the actual configuration of the environment of the $\sigma_{0}$-system. In other words, the indicator function of 
the event $\left\{\sigma=\sigma_{0}\right\}$, the function $\mathbf{1}_{\left\{\sigma=\sigma_{0}\right\}}$ which is one if $Q \in\left\{\sigma=\sigma_{0}\right\}$ and is zero otherwise, must be a functional of the actual configuration of the environment of the $\sigma_{0}$-system:

$$
\mathbf{1}_{\left\{\sigma=\sigma_{0}\right\}}=F\left[Y_{\sigma_{0}}\right]
$$

We call a pair $\sigma=(\pi, T)$ satisfying (5.2) a random system. Note that, owing to (5.2), random systems are nonrandom relative to their environment. It is for this reason that we usually fail to notice that our systems are random: relative to "ourselves," which we naturally don't think of as random, they are completely determined. The condition (5.2) fits nicely with the notion of the wave function of a subsystem, as expressed, e.g., by (4.1); moreover it allows a natural extension of the fundamental conditional probability formula (5.1) to random systems (see [7]): for any random system $\sigma$

$$
\mathbf{P}^{\Psi_{0}}\left(X_{\sigma} \in d x \mid Y_{\sigma}, \sigma\right)=\left|\psi_{\sigma}(x)\right|^{2} d x
$$

where $\psi_{\sigma}$ is the (effective or conditional) wave function of the random system $\sigma$. (Given the initial universal configuration $Q, \psi_{\sigma}$ is the wave function at time $T(Q)$ of the system defined by $\pi(Q))$. The formula (5.3) can in a sense be regarded as the most compact expression of the entire quantum formalism. ${ }^{1}$

In order to describe the repetion of similar experiments, performed at different places and times, in a single sample of the the universe we consider a sequence $\sigma_{i}=\left(\pi_{i}, T_{i}\right), i=1, \ldots, M$, of random systems such that the requirement that the $i$-th system have wave function $\psi$ forms part of the basis of selection for this

\footnotetext{
${ }^{1}$ The reader familiar with the theory of stochastic processes should note the similarity between (5.2) and (5.3) on the one hand, and the notions of stopping time and the strong Markov property from Markov process theory on the other. Indeed, (5.1) can be regarded as a kind of Markov property, in relation to which (5.3) then becomes a strong Markov property.
} 
system, i.e., the $\sigma_{i}$ satisfy the condition: $\psi_{\sigma_{i}}=\psi$. Moreover, we demand that the following measurability condition be satisfied: If $T_{\sigma_{i}}<T_{\sigma_{j}}$ the information about the measured value of $X_{\sigma_{i}}$ must be grounded in the environment of $X_{\sigma_{j}}$; in other words, (with probability 1) for all $T_{\sigma_{i}}<T_{\sigma_{j}}, X_{\sigma_{i}}$ must be a functional of $Y_{\sigma_{j}}$,

$$
X_{\sigma_{i}}=G_{i j}\left[Y_{\sigma_{j}}\right] \quad \text { whenever } T_{\sigma_{i}}<T_{\sigma_{j}} .
$$

Under these conditions $X_{\sigma_{1}}, \ldots, X_{\sigma_{M}}$ are independent, with each $X_{\sigma_{i}}$ having distribution given by $|\psi|^{2}$, see [7]. Thus, by the weak law of large numbers, for large $M$, it follows that for our sequence of experiments typical initial configurations yield empirical statistics governed by Born's statistical law.

Such a result holds, without further assumptions, when typicality is expressed by the measure $\mathbf{P}^{\Psi_{0}}$, but also, and more importantly, when $\mathbf{P}^{\Psi_{0}}$ is replaced by a conditional measure $\mathbf{P}^{\Psi_{0}, \mathcal{M}}(d Q)=\mathbf{P}^{\Psi_{0}}(d Q \mid \mathcal{M})$, where $\mathcal{M} \subset \mathcal{Q}$, provided the random systems $\sigma_{i}$ under consideration satisfy the functional relation

$$
\mathbf{1}_{\mathcal{M}}=J_{i}\left[Y_{\sigma_{i}}, \sigma_{i}\right]
$$

Indeed, conditioning on the event $\mathcal{M}$ takes into account any kind of "prior" informationalways present - reflecting the "macroscopic state" $\mathcal{M}$ at a time prior to all experiments.

We emphasize that the assumptions (5.4) and (5.5) are physically minimal. They demand merely that "facts" about results and initial experimental conditions not be forgotten. Moreover it is not hard to see that if these conditions are relaxed (e.g., by allowing the possibility of "forgetting" the results in a selective manner), the results should not be expected to agree with the predictions of the quantum formalism. 


\section{UnCERTAINTY AND PROBABILITY IN BOHMIAN MEChaniCS}

The most remarkable consequence of our analysis is absolute uncertainty: Since any (environmentally-based) selection criterion, whatever it may be, can be incorporated into the definition of our random systems - as part of the basis for their selection - it follows that no such criterion can be regarded as reflecting any information, beyond $|\psi|^{2}$, about the configurations of these systems when their wave function is $\psi$. Thus, in a universe governed by Bohmian mechanics it is in principle impossible to know more about the configuration of a subsystem than what is expressed by Born's law. ${ }^{2}$

We remark that the general structure of our random system analysis does not depend upon the detailed structure of Bohmian mechanics: any modeling of the universe in terms of a deterministic dynamical system would demand a similar analysis. In order to get a handle on the hallmark of Bohmian mechanics which leads to absolute uncertainty, we may consider a very general dynamical framework where typicality is expressed by a (nonstationary) measure $\mu$ on the space of initial conditions and $X$ and $Y$ denote the "microstates" respectively of a random system $\sigma$ and its environment.

Also in this general case we have that an "experimenter" can achieve knowledge of the $x$-system only if his information about this system is grounded in its environment (of which the experimenter must be a part). However, even if the experimenter somehow has access to the full microscopic environment (grossly more than what could be possibe for any realistic experimenter!), the state of the $x$ system will appear to be random and this randomness will be governed by the

\footnotetext{
${ }^{2}$ The reader may be disturbed by the fact that since only the absolute value of $\psi$ determines the statistics for the configuration, measuring configurations may not inform us about $\psi$. If so, he should recall that the phase of $\psi$ guides the time evolution of the configurations.
} 
conditional distribution $\mu(d X \mid Y)$.

This conditional distribution will in general be given by a (density) function $f(x, y)$ which depends upon the system $\sigma$ and, of course, upon $\mu$, but which is however to a large extent unknown. In the quantum case under consideration in this paper $f(x, y)$ is given by (5.3) and thus depends upon $y$ through the (effective or conditional) wave function of the $x$-system, an "object" which has dynamical significance. In general, however, $f(x, y)$ need play no dynamical role vis a vis the future behavior of the $x$-system. A situation of this kind occurs, for instance, in classical mechanics, where, moreover, the evolution of macrostates (given, e.g., by (2.4), regarded as a conditional probability formula) is not "equivariantly" related to the dynamical law governing the evolution of microstates (see below and footnote 3$)$.

Thus, what lends substance to the "absolute uncertainty" in Bohmian mechanicsand justifies our use of that phrase - is the fact that there $f(x, y)$ is a functional of $\psi$, an object whose significance is primarly dynamical. The detailed character of this dynamical aspect is such that a wave function with narrow support quickly spreads, owing to the dispersion in Schrödinger's equation, to one with broad support, a change which generates a similar change in the distribution of the configuration and thus to an unavoidable uncertainty in future configurations, and hence in the "effective velocity." Absolute uncertainty is then in precise agreement with Heisenberg's uncertainty principle [9]. (Note, however, that while Heisenberg used uncertainty to argue for the meaninglessness of particle trajectories, here quantum uncertainty arises as a simple consequence of the existence of trajectories.)

Note, moreover, that-owing to the critical dependence of the (conditional or effective) wave function of a subsystem upon its environment, where all knowl- 
edge is grounded - the dynamical role of the conditional distribution $f(x, y)$ within Bohmian mechanics illuminates another hallmark of the Copenhagen interpretation: the epistemological component, the appeal to the observer, with which the wave function has traditionally been entangled.

We arrive then at the conclusion that local quantum randomness - as described by Born's statistical law — and quantum uncertainty - the impossibility of obtaining information about the actual configuration more detailed than what is given by the quantum equilibrium distribution - are merely an expression of quantum equilibrium, a global configurational equilibrium relative to the universal wave function $\Psi$. The physical significance of quantum equilibrium is thus as a measure of typicality and the ultimate justification of Born's statistical law is in terms of the statistical behavior arising from a typical initial global configuration.

More generally, for any theory with probablistic content, particularly one describing a relativistic universe, we arrive at a similar conclusion: Once we recognize that there is but one world (of relevance to us), only one actual space-time history, we must also recognize that the ultimate meaning of probability, insofar as it is employed in the formulation of the predictions of the theory, must be in terms of a specification of typicality — one such that theoretically predicted empirical distributions are typical. In fact, when all is said and done, the physical import of the theory must arise from its provision of such a notion of typical space-time histories, presumably specified via a probability distribution on the set of all (kinematically) possible histories.

One may argue that all a theory should do is to provide a notion of typical "macroscopic events," for example by extracting from the standard quantum for- 
malism a "quasiclassical domain of familiar experience" as suggested by Gell-Mann and Hartle [11]. But as we noted in the introduction, such attempts will naturally suffer from the imprecision and ambiguity inherent in any reference to the "macroscopic," as well as from failing to ground the notion of typicality. Accordingly we note also that insofar as nonrelativistic quantum theory is concerned a significant difference between Bohmian mechanics and the proposal of GMH is that the latter defines a research program, while the former is an already existing, and sharply formulated, physical theory.

We wish to emphasize this byproduct of our analysis, that quite aside from the relevance of this analysis to the interpretation of quantum theory, we find a clarification and illumination of the meaning and role of probability in a deterministic universe. It is tempting to say that Bohmian mechanics offers the first natural complete explanation of the emergence of statistical laws from a deterministic mechanics, by overcoming those difficulties which may arise in the framework of a different mechanical theory, such as, for example, Newtonian mechanics. ${ }^{3}$

Moreover, the analysis of Bohmian mechanics presented here is relevant to the problem of the interpretation and application of quantum theory in cosmology, specifically, to the problem of the significance of $\rho=|\psi|^{2}$ on the cosmological level - where there is nothing outside of the system to perform the measurements from which $\rho=|\psi|^{2}$ derives its very meaning in orthodox quantum theory.

\footnotetext{
${ }^{3}$ Krylov has made an extensive and penetrating analysis of such difficulties. A (rough) summary of his point was given by Krylov himself: "The impossibility of interpreting the probabilistic laws of statistical mechanics within the framework of the classical theory stems, in fact, from the absence of a necessary connection between the fact of the system being in a given phase space region (for example, being in a given macroscopic state) and a certain law of distribution of microstates within that region; in other words, it stems from the absence of a necessary relation between the fact of the system being in a given macroscopic state and a certain distribution of probabilities in the further development of the process that conforms to the laws of statistics and kinetics [14]." It should be clear from our presentation that equivariance guarantees the existence for Bohmian mechanics of that necessary relation absent in classical mechanics.
} 
Finally we wish to stress that our random system analysis illuminates the flexibility of Bohmian mechanics: It illustrates how joint probabilities as predicted by the quantum formalism, even for configurations, may arise from measurement and bear little resemblance to the probabilities for unmeasured quantities. And our analysis highlights the mathematical features which make this possible. This flexibility could be quite important for achieving an understanding of the relativistic domain, where it may happen that quantum equilibrium prevails only on special space-time surfaces (see [8] and [4]). Our random system multitime analysis illustrates how this need entail no genuine obstacle to obtaining the quantum formalism. ${ }^{4}$

\section{ERgodic Properties of Bohmian MeCHANiCS}

From a dynamical systems perspective, it would appear natural to attempt to justify Born's statistical law directly — without appealing to any cosmological analysis-by using, for example, such notions as "convergence to equilibrium," "mixing," or "ergodicity," suitably generalized.

However, it might seem that Bohmian mechanics rather trivially fails to possess good ergodic properties, if one considers the motion arising from the standard energy eigenstates of familiar systems. However, quantum systems attain such simple wave functions only through complex interactions, for example with an apparatus during a measurement or preparation procedure, during which time they are not governed by a simple wave function. Thus the question of the ergodic properties of Bohmian mechanics refers to the motion under generic, more complex, wave functions.

\footnotetext{
${ }^{4}$ Our argument here of course involved the natural hypersurfaces given by $\{t=$ const. $\}$, but the only feature of these surfaces critical to our analysis was the validity of quantum equilibrium, or, more precisely, of the fundamental conditional probability formula (5.3).
} 
Be that as it may, we have shown that establishing such properties is neither necessary nor sufficient for the purpose of founding the statistical character of a deterministic theory.

Conventional wisdom to the contrary notwithstanding, the problem of the rigorous justification, from first principles, of the use of the "standard ensembles," i.e., of the derivation of randomness governed by detailed probabilities, is far more difficult for classical thermodynamic equilibrium than for quantum theory! How can this be? How is it possible so easily to derive the local equilibrium behavior governed by Born's statistical law from first principles (i.e., from Bohmian mechanics), while the corresponding result for thermodynamics - the rigorous derivation of the Gibbs postulate from first principles - is so very difficult? The answer, we believe, is that "pure equilibrium" is easy, while nonequilibrium, even a little bit, is hard. In our nonequilibrium universe, systems which happen to be in thermodynamic equilibrium are surrounded by, and arose from, (thermodynamic) nonequilibrium. Thus with thermodynamic equilibrium we are dealing with islands of equilibrium in a sea of nonequilibrium. But with quantum equilibrium we are in effect dealing with a global equilibrium, albeit relative to the wave function.

A key aspect of equilibrium is, of course, stationarity - or equivariance. But how can this be sufficient for our purposes? Mere stationarity is not normally sufficient in a dynamical system analysis to conclude that typical behavior embodies randomness governed by the stationary distribution. Such "almost everywhere"-type assertions usually require the ergodicity of the dynamics. Why did we not find it necessary to establish some sort of ergodicity?

The answer lies in another critical aspect of the notion of equilibrium, arising from the fact that we are concerned with "large systems," with the thermody- 
namic limit as it were. In equilibrium, whether quantum or thermodynamic, most configurations or phase points are "macroscopically similar": quantities given by suitable spatial averages - e.g., density, energy density, or velocity fluctuations for thermodynamic equilibrium, and empirical correlations for quantum equilibriumare more or less constant over the state space, in a sense defined by the equilibrium distribution. To say that a system is in equilibrium is then to say that its configuration or phase point is typical, in the sense that the values of these spatial averages are typical.

Now while the individual subsystems with which we have been concerned may be microscopic, our analysis, in fact, is effectively a "large-system analysis." This is implicit, for example, in our measurability conditions (5.4) and (5.5), which are plausible only for a universe having a large number of degrees of freedom. Thus, just as for a system already in thermodynamic equilibrium, we have no need for the ergodicity of the dynamics - but only for "stationarity" - since the kind of behavior we wish to establish occurs for a huge set of initial configurations, the "overwhelming majority."

When all is said and done, it might be said that we have in fact established for Bohmian mechanics a kind of effective Bernoulliness, and hence an effective ergodicity.

\section{ACKNOWLEDGEMENTS}

One of us (N.Z.) wishes to dedicate this paper to Professor Asim O. Barut, from whom he learned how to move in theoretical physics without prejudices, in particular the ones hidden in the quantum orthodoxy. We are grateful to G. Eyink for valuable discussions. 


\section{REFERENCES}

1. J. S. Bell, Beables for Quantum Field Theory, CERN-TH 4035/84, reprinted in [2].

2. J. S. Bell, Speakable and unspeakable in quantum mechanics, Cambridge University Press, Cambridge, 1987.

3. J. S. Bell, Against measurement, Physics World 3 (1990), 33-40.

4. K. Berndl, Doctoral Thesis, in preparation.

5. D. Bohm, A suggested interpretation of the quantum theory in terms of "hidden variables", Physical Review 85 (1952), 166-193.

6. D. Bohm, B.J. Hiley and P.N. Kaloyerou, An Ontological Basis for Quantum Theory.II A causal interpetation of quantum fields, Physics Reports 144 (1987), 349-375.

7. D. Dürr, S. Goldstein, and N. Zanghí, Quantum Equilibrium and the Origin of Absolute Uncertainty, J. Stat. Phys. 67 (1992), 843-907.

8. D. Dürr, S. Goldstein, and N. Zanghí, On a Realistic Theory for Quantum Physics, Stochastic Processes, Geometry and Physics (S. Albeverio, G. Casati, U. Cattaneo, D. Merlini, R. Mortesi, eds.), World Scientific, Singapore, 1990, pp. 374-391.

9. D. Dürr, S. Goldstein, and N. Zanghí, On the role of operators in quantum theory, in preparation.

10. H. Everett, 'Relative State' Formulation of Quantum Mechanics, Rev. Mod. Phys. Phys. 29 (1957), 454-462.

11. M. Gell-Mann and J.B. Hartle, Quantum mechanics in the light of quantum cosmology, Complexity, Entropy, and the Physics of Information (W. Zurek, eds.), Adison-Wesley, Reading, 1990, pp. 425-458.

12. G. C. Ghirardi, A.Rimini, and T. Weber, Unified dynamics for microscopic and macroscopic systems, Phyis. Rev. D 34 (1986), 470-491.

13. W. Heisenberg, Physics and Beyond, Harper and Row, New York, 1971, p. 63.

14. N. S. Krylov, Works on the Foundations of Statistical Mechanics, Princeton University Press, Princeton, N.J., 1979.

15. R. Penrose, The Emperor's New Mind, Oxford University Press, New York and Oxford, 1989. 